\title{
The dynamics of cluster substructure
}

\section{Stuart P. D. Gill, Alexander Knebe and Brad K. Gibson}

Centre for Astrophysics \& Supercomputing, Swinburne University, Mail \#31, P.O. Box 218, Hawthorn, VIC 3122, Australia email: sgill@astro.swin.edu.au

\begin{abstract}
To understand cluster formation and evolution it is important to understand the evolution of cluster substructure. The work described here focuses upon a set of fully selfconsistent numerical simulations within a $\Lambda \mathrm{CDM}$ cosmology. Within the simulations we follow in detail the temporal and spatial properties of individually tracked satellite galaxies. The host galaxy clusters were chosen to sample a variety of formation histories, ages, and triaxialities. Despite their obvious differences, we find striking similarities within the associated substructure populations. Namely, the satellite galaxy orbital distributions are indistinguishable between the galaxy clusters.
\end{abstract}

\section{Introduction}

There has been growing convergence to a "concordance model" for cosmological structure formation, with a "standard" $\Lambda \mathrm{CDM}$ universe comprised of $28 \%$ dark matter, $68 \%$ dark energy, and luminous baryonic matter (i.e. galaxies, stars, gas, and dust) at a mere $4 \%$ (cf. Spergel et al. 2003). In such a universe structures form bottom-up with small objects forming first that subsequently merge to form progressively larger objects (e.g. White \& Reese 1978; Davis et al. 1985). Hence, galaxies and galaxy clusters are constantly fed by smaller dark halos that begin to orbit within the encompassing dark matter host. If this model is correct, it is important to understand the orbital evolution of these objects and investigate how they accrete onto the host halo. The work presented here is based upon a series of high-resolution numerical simulations within said concordance model. The analysis focuses on the detailed orbital parameters of satellite galaxies and internal properties.

The first fully self-consistent simulations targeting the subject were performed by Tormen et al. (1997, 1998). Both studies were excellent efforts. However they lacked the temporal, spatial, and mass resolution required to explore a wide range of environmental effects. Unable to follow the satellite distribution within the host's virial radius, satellites were instead tracked only up to and including the point of "accretion". This allowed an analysis of the infall pattern, rather than the orbital evolution of the satellites. Ghinga et al. (1998) also investigated the dynamics of satellite galaxies in live dark matter host halos. Although greatly increasing the mass and spatial resolution, they still lacked the temporal resolution to explicitly track the satellite orbits, rather they approximated the orbits using a spherical static potential again. In these proceedings we will present a series of simulations with the required resolution to follow the satellites even within the very central regions of the host potential $(\geqslant 5-10 \%$ of the virial radius) and the time resolution to resolve the satellite dynamics with unprecedented accuracy ( $\Delta t \approx 170$ Myrs).

\section{Simulations and halo identification}

The following section outlines our set of eight high-resolution galaxy clusters each consisting of order more than a million dark matter particles and the methods used to 


\begin{tabular}{cccccc}
\hline Halo & $R_{\text {vir }}$ & $M_{\text {vir }}$ & $z_{\text {form }}$ & age & $N_{\text {sat }}\left(<R_{\text {vir }}\right)$ \\
\hline$\# 1$ & 1.34 & 2.87 & 1.16 & 8.30 & 158 \\
$\# 2$ & 1.06 & 1.42 & 0.96 & 7.55 & 63 \\
$\# 3$ & 1.08 & 1.48 & 0.87 & 7.16 & 87 \\
$\# 4$ & 0.98 & 1.10 & 0.85 & 7.07 & 57 \\
$\# 5$ & 1.35 & 2.91 & 0.65 & 6.01 & 175 \\
$\# 6$ & 1.05 & 1.37 & 0.65 & 6.01 & 85 \\
$\# 7$ & 1.01 & 1.21 & 0.43 & 4.52 & 59 \\
$\# 8$ & 1.38 & 3.08 & 0.30 & 3.42 & 251 \\
\hline
\end{tabular}

Table 1. Properties of the eight dark matter host halos. Distances are measured in $h^{-1} \mathrm{Mpc}$, velocities in $\mathrm{km} \mathrm{s}^{-1}$, masses in $10^{14} h^{-1} \mathrm{M}_{\odot}$, and the age in Gyr. We applied a mass-cut of $M>2 \times 10^{10} h^{-1} \mathrm{M}_{\odot}(100$ particles $)$ which explains the rather 'low' number for $N_{\text {sat }}\left(<R_{\text {vir }}\right)$.

analyse them. These clusters formed in dissipationless $N$-body simulations characterised by a $\Lambda$ CDM cosmology $\left(\Omega_{0}=0.3, \Omega_{\lambda}=0.7, \Omega_{b} h^{2}=0.04, h=0.7, \sigma_{8}=0.9\right)$. The runs have a mass resolution of $m_{p}=1.6 \times 10^{8} h^{-1} \mathrm{M}_{\odot}$ and achieved a force resolution of $\approx 2 h^{-1} \mathrm{kpc}$ allowing us to resolve the host halos down to about the central $0.25 \%$ of their virial radii $R_{\text {vir }}$. High temporal information was required to determine the orbital development of the satellites. We therefore stored 17 outputs from $z=2.5$ to $z=0.5$ equally spaced with $\Delta t \approx 0.35$ Gyr. From $z=0.5$ to $z=0$ we have 30 outputs spaced $\Delta t \approx 0.17$ Gyr. A summary of the eight host halos, is presented in Table 1 .

These simulations were analysed using two new methods for identifying gravitationally bound objects, MHF (MLAPM Halo Finder) and MHT (MLAPM Halo Tracker). A more elaborate description of this technique can be found elsewhere (Gill, Knebe \& Gibson 2004). The finder (MHF) utilizes the adaptive meshes of the open source $N$-body code MLAPM $\dagger$ (Knebe, Green \& Binney 2001). The adaptive meshes of MLAPM are recursive: refined regions can also be refined. This creates a hierarchy of refinement meshes of different resolutions covering regions of interest. The refinements are created cell by cell they are not constrained to have a rectangular (or any other) shape. Thus, the grid structure naturally surrounds the (satellite) galaxies as they manifest themselves as over-densities in the underlying background field. The tracker (MHT) takes an initial output of MHF and then tracks the particles that were a member of that satellite at later times.

\section{The orbital parameters of cluster substructure}

In this section we investigate the orbital parameters of the cluster substructure. Figure 1 on the LHS plots the satellite eccentricity against pericentre normalised by the virial radius of the host. The crosses represent the satellites that survived until $z=0$ while the diamonds represent the disrupted satellites. The orbital eccentricity of the substructure is defined to be:

$$
e=1-\frac{p}{a}
$$

where the pericentre $p$ and apocentre $a$ distances are the last measured in the satellite's orbit.

The interesting feature of this figure is that there seems to be a distinct population of satellites with an extremely tight anti-correlation between eccentricities $e \approx 0.6-0.9$ and pericentres $p \approx 0.3-0.8$. Upon detailed investigation these satellites were identified to have just completed one orbit and hence entering the halo for the first time.

$\dagger$ MLAPM can be downloaded from the web page http://astronomy.swin.edu.au/MLAPM 

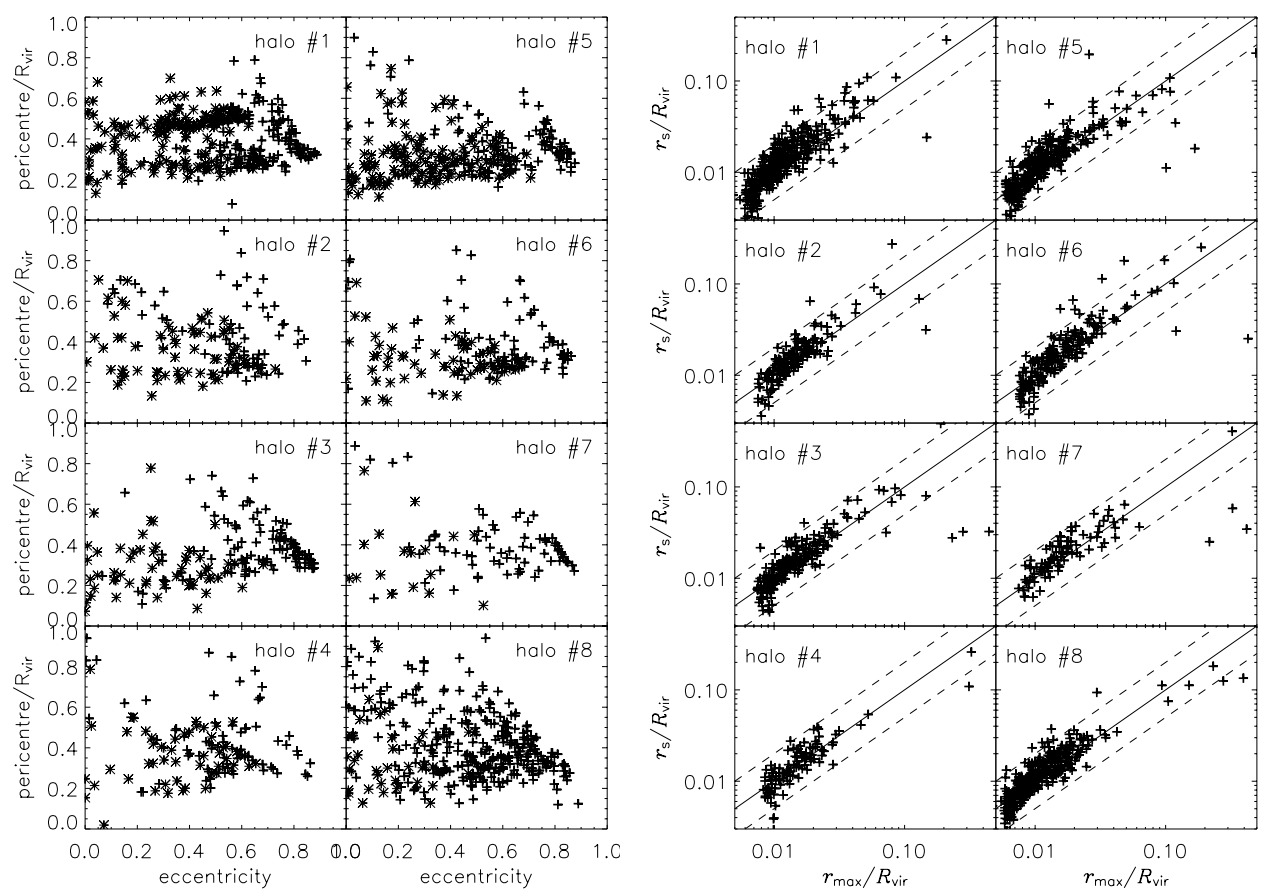

Figure 1. LHS: We plot the satellite eccentricity against pericentre normalised by the virial radius of the host. The crosses represent the satellites that survived until $z=0$ while the stars represent the disrupted satellites. RHS: We plot the relation between scale radius $r_{s}$ defined in eq. (4.1) and the position of the maximum of the rotation curve. Both of these values are normalised by the hosts virial radius.

Morevoer, using this definition for eccentricity the peak in the orbital eccentricity distribution for the eight halos has an average of $\left\langle e_{0}\right\rangle=0.61$ with an average standard deviation of $\langle\sigma\rangle=0.19$. The disrupted satellites, however, have quite a different distribution with the peak eccentricity at around $\left\langle e_{0}^{d}\right\rangle=0.34$ and a dispersion of $\left\langle\sigma^{d}\right\rangle=0.16$. The pericentre distribution's maximum peak lies at $35 \%$ of the virial radius for live satellites with a mean dispersion of $\langle\sigma\rangle=0.12$ as opposed to $31 \%$ of $R_{\text {vir }}$ for the disrupted ones with a dispersion of $\left\langle\sigma^{d}\right\rangle=0.11$.

There seems to be a lack in correlation with mass, age, environment and richness of the host halos and their substructure orbital parameters. However, there is a difference between "live" and "disrupted" satellites. Disrupted satellites are on more circular orbits. Even though they orbit with similar pericentres, the circular nature of their orbit means that the satellites spend longer times in the deeper regions of the potential well, hence, experiencing stronger tidal forces for longer periods, thus being disrupted.

We like to stress that for the satellites that survived until $z=0$ more than $70 \%$ of the satellites had at least one full orbit with some having as many as four orbits.

\section{Density profiles of substructure halos}

For field galaxies in cosmological simulations there is the well know relationship between $r_{\max }$, the point where the rotation curve of the satellite galaxy peaks and the scale radius $r_{s}$ (as defined in eq. 4.1, Bullock 2001). In this section we aim to verify this relation for substructure halos, which on analytical grounds should be of the order $r_{\max } \approx r_{s}$. 
To verify this analytical relation we rather use the cumulative density profile $\rho(<r)$ than the differential $\rho(r)$; for (sub-)halos containing only few particles this leads to a smoother profile and hence a more stable fit to an analytical function:

$$
\rho^{\operatorname{cum}}(r)=\frac{M(<r)}{4 \pi r^{3} / 3}=\frac{\rho_{s} r_{s}^{3}}{r\left(r_{s}+r\right)^{2}}
$$

where $r_{s}$ is our scale radius used to calculate the concentration $c=r_{\text {vir }} / r_{s}$.

Navarro et al. (1997) used the differential density profile

$$
\rho^{\mathrm{NFW}}(r)=\frac{d M(r)}{d V}=\frac{\rho_{s}^{\mathrm{NFW}} R_{s}^{3}}{r\left(R_{s}+r\right)^{2}}
$$

where $r_{s}$ is again used to define the concentration $c_{\mathrm{NFW}}=r_{\mathrm{vir}} / R_{s}$ of the halo.

We are interested in a relation between our scale radius $r_{s}$ and the maximum of the circular rotation curve $r_{\max }$ as presented for our subhalo populations in Figure 1. Therefore using

$$
v_{\text {circ }}^{2}=\frac{G M(<r)}{r}
$$

together with eq. (4.1) gives

$$
v_{\text {circ }}^{2}=\frac{4 \pi G r \rho_{s} r_{s}^{3}}{3\left(r_{s}+r\right)^{2}} .
$$

We obtain the maximum of $v_{\text {circ }}$ by differentiating it with respects to $r$

$$
\frac{d v_{\text {circ }}}{d r}=\frac{1}{2 v_{\text {circ }}} \frac{4 \pi G r_{s}^{3}}{3\left(r_{s}+r\right)^{2}}\left(1-\frac{2 r}{r_{s}+r}\right)
$$

and setting it to zero. Simple algebra then reveals the relation

$$
r_{s}=r_{\max }
$$

To verify this simple relation we plot $r_{s}$ versus $r_{\max }$ on the RHS of Figure 1 . We find that this relation holds for all our satellite galaxies with very little scatter. The solid line is the one-to-one relation with the dashed lines showing $r_{s}=2 r_{\max }$ (upper) and $r_{s}=0.5 r_{\max }$ (lower).

\section{Summary}

We used a set of eight high-resolution cosmological simulations to investigate and quantify the orbital parameters of satellite dark matter galaxies within host cluster systems. We found that even though the eight dark matter host halos were quite different with different accretion histories, their respective satellite population showed remarkably similar properties. For the satellites that survived until $z=0$ the average orbital eccentricity of the satellites was found to be $e \approx 0.61$ with as little scatter as $\sigma \approx 0.19$. Moreover, the average pericentre distance of the satellites was $p \sim 35 \%$ of $R_{\mathrm{vir}}$ for all halos with again very little scatter about the mean i.e. $\sigma \approx 0.12$. For the disrupted satellite population at $z=0$ we found a very similar pericentre distribution; however, their eccentricity distribution was considerably lower, $e^{d} \approx 0.34$. This implies that the disrupted satellites spend longer times in the deeper regions of the potential well, hence, experiencing stronger tidal forces for longer periods, thus being disrupted quicker.

Finally we verified the relationship for substructure galaxies between the maximum of their rotation curves and the scale radius $r_{s}$ as defined by the NFW profile. 


\section{Acknowledgements}

The simulations presented in this paper were carried out on the Beowulf cluster at the Centre for Astrophysics \& Supercomputing, Swinburne University.

\section{References}

Bullock J., Kolatt T.S., Sigad Y., Somerville R.S., Kravtsov A.V., Klypin A.A., Primack J.R., Dekel A., 2001 MNRAS 559, 321

Davis M., Efstathiou G., Frenk C. S., \& White S. D. M., 1985 ApJ 292, 371

Ghigna, S., Moore, B., Governato, F., Lake, G., Quinn, T., Stadel, J. 1998 MNRAS 300, 146

Gill S.P.D., Knebe A., Gibson B.K., 2004, MNRAS in press

Knebe A., Green A., Binney J., 2001 MNRAS 325, 845

Navarro J., Frenk C.S., White S.D.M., 1997 ApJ 490, 493

Spergel D. N., Verde L., Peiris H. V., Komatsu E., Nolta M. R., Bennett C. L., Halpern M., Hinshaw G., Jarosik N., Kogut A., Limon M., Meyer S. S., Page L., Tucker G. S., Weiland J. L., Wollack E., Wright E. L., 2003 ApJ Suppl. 148, 175

Tormen G., Bouchet F., White S. D. M., 1997 MNRAS 286, 865

Tormen G., Diaferio A., Syer D., 1998 MNRAS 299, 728

White S. D. M., \& Rees M., 1978 MNRAS 183, 341 